Appendix 2. 40Ar/39Ar analytical data.

ID 40Ar/39Ar 37Ar/39Ar 36Ar/39Ar 39ArK K/Ca

(x 10-3) $\times 10-15 \mathrm{~mol})$

$\mathrm{Ca}$

(1)

\section{Tuff of Candelaria}

\begin{tabular}{|c|c|c|c|c|c|c|c|c|c|}
\hline & TT10 & iotite, $\mathrm{J}=$ & 009425 & $0.07 \%, D=1$ & $063 \pm 0.0$ & NM-174F & $\mathrm{Lab \# =5}$ & & \\
\hline \# & 03A & 193.487 & 2.223 & 832.790 & 0.014 & 0.230 & -27.091 & -91.548 & 23.344 \\
\hline \# & $01 \mathrm{~A}$ & 431.783 & 1.177 & 1517.041 & 0.008 & 0.434 & -3.799 & -28.135 & 37.741 \\
\hline \# & $02 \mathrm{~A}$ & 189.506 & 1.372 & 683.452 & 0.017 & 0.372 & -6.512 & -21.125 & 19.693 \\
\hline \# & $10 \mathrm{~A}$ & 521.253 & 0.090 & 1739.584 & 0.796 & 5.661 & 1.384 & 12.223 & 4.284 \\
\hline \# & $04 \mathrm{~A}$ & 113.798 & 0.131 & 350.051 & 0.270 & 3.902 & 9.111 & 17.545 & 2.265 \\
\hline \# & $05 A$ & 315.869 & 0.039 & 1031.285 & 1.272 & 13.207 & 3.523 & 18.822 & 2.502 \\
\hline \# & 08A & 605.324 & 0.043 & 2009.916 & 1.223 & 11.848 & 1.883 & 19.276 & 4.528 \\
\hline \# & 07A & 371.748 & 0.136 & 1218.872 & 0.669 & 3.755 & 3.116 & 19.588 & 3.348 \\
\hline \# & 09A & 440.219 & 0.126 & 1448.255 & 1.005 & 4.040 & 2.787 & 20.744 & 3.418 \\
\hline \# & 09B & 16.306 & 0.035 & 11.328 & 2.192 & 14.646 & 79.490 & 21.905 & 0.286 \\
\hline \# & $10 \mathrm{~B}$ & 22.101 & 0.035 & 30.374 & 1.306 & 14.450 & 59.403 & 22.186 & 0.375 \\
\hline \# & 02B & 14.249 & 0.048 & 2.874 & 3.977 & 10.688 & 94.068 & 22.648 & 0.218 \\
\hline \# & 08B & 26.538 & 0.071 & 44.177 & 2.465 & 7.221 & 50.832 & 22.793 & 0.368 \\
\hline & 01B & 15.892 & 0.046 & 7.853 & 7.415 & 11.174 & 85.422 & 22.936 & 0.203 \\
\hline & 05B & 16.526 & 0.016 & 9.845 & 5.532 & 32.144 & 82.405 & 23.008 & 0.215 \\
\hline \# & 06A & 53.403 & 0.999 & 134.694 & 0.048 & 0.511 & 25.623 & 23.133 & 5.849 \\
\hline & 07B & 16.308 & 0.055 & 8.735 & 4.444 & 9.342 & 84.200 & 23.198 & 0.221 \\
\hline & 03B & 14.517 & 0.025 & 2.623 & 6.599 & 20.043 & 94.675 & 23.219 & 0.201 \\
\hline & 04B & 14.288 & 0.029 & 1.764 & 20.853 & 17.409 & 96.368 & 23.262 & 0.190 \\
\hline & 06B & 14.583 & 0.029 & 2.616 & 5.354 & 17.657 & 94.716 & 23.334 & 0.202 \\
\hline & Mear & $g e \pm 2 s$ & $n=6$ & USWD $=0.57$ & & $18.0 \pm 16.2$ & & 23.164 & 0.170 \\
\hline
\end{tabular}

\section{Candelaria Junction Tuff}

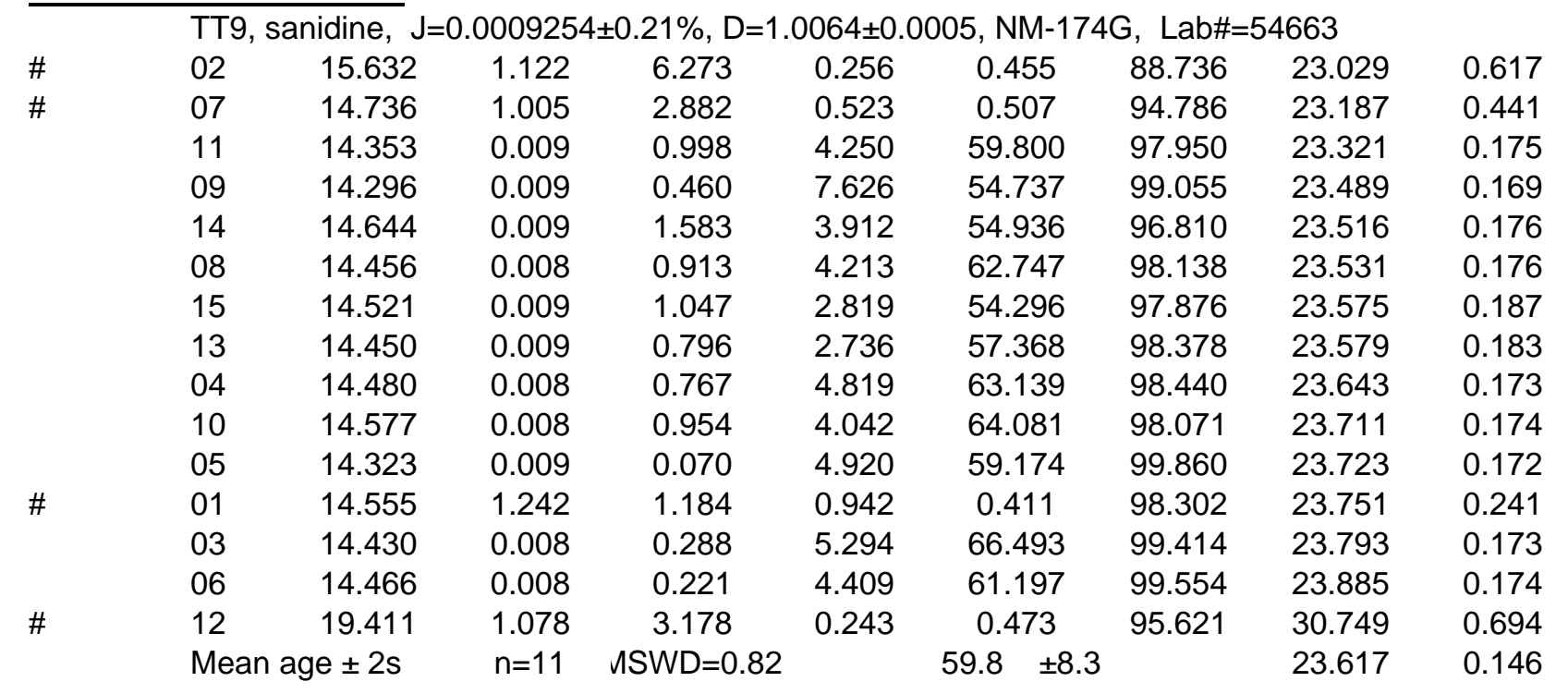




\begin{tabular}{|c|c|c|c|c|c|c|c|c|c|}
\hline & \multicolumn{9}{|c|}{ TT9, sanidine, $J=0.0010107 \pm 0.07 \%, D=1.0064 \pm 0.0005, N M-174 F, \quad$ Lab\#=54656 } \\
\hline \# & 02 & 13.508 & 1.057 & 3.622 & 1.323 & 0.483 & 92.725 & 22.712 & 0.270 \\
\hline \# & 12 & 13.816 & 1.185 & 4.006 & 0.426 & 0.431 & 92.141 & 23.082 & 0.571 \\
\hline \multirow[t]{12}{*}{ \# } & 11 & 13.602 & 1.499 & 3.227 & 0.436 & 0.340 & 93.901 & 23.165 & 0.537 \\
\hline & 13 & 13.807 & 0.013 & 3.150 & 2.602 & 39.668 & 93.266 & 23.328 & 0.224 \\
\hline & 08 & 13.058 & 0.008 & 0.437 & 4.424 & 61.874 & 99.015 & 23.422 & 0.209 \\
\hline & 07 & 14.963 & 0.007 & 6.822 & 12.171 & 70.519 & 86.531 & 23.455 & 0.204 \\
\hline & 09 & 13.309 & 0.007 & 1.082 & 8.450 & 71.833 & 97.602 & 23.532 & 0.203 \\
\hline & 06 & 13.180 & 0.271 & 0.693 & 5.362 & 1.885 & 98.617 & 23.550 & 0.206 \\
\hline & 03 & 13.283 & 0.008 & 0.856 & 8.681 & 63.903 & 98.100 & 23.605 & 0.203 \\
\hline & 04 & 13.082 & 0.008 & -0.003 & 4.653 & 65.902 & 100.012 & 23.701 & 0.205 \\
\hline & 14 & 13.139 & 0.005 & 0.180 & 4.123 & 102.995 & 99.598 & 23.705 & 0.210 \\
\hline & 10 & 13.252 & 0.009 & 0.463 & 6.113 & 54.750 & 98.974 & 23.758 & 0.205 \\
\hline & 01 & 13.152 & 0.009 & 0.118 & 13.093 & 59.076 & 99.741 & 23.762 & 0.202 \\
\hline & 15 & 13.198 & 0.004 & 0.109 & 2.762 & 144.767 & 99.757 & 23.849 & 0.212 \\
\hline \multirow[t]{3}{*}{ \# } & 05 & 13.489 & 1.195 & 1.237 & 0.472 & 0.427 & 98.024 & 23.969 & 0.486 \\
\hline & \multicolumn{2}{|c|}{ Mean age $\pm 2 s$} & $n=11$ & \multicolumn{2}{|c|}{ USWD $=0.60$} & $67.0 \pm 71.0$ & \pm 71.0 & 23.609 & 0.129 \\
\hline & \multicolumn{9}{|c|}{ TT2ca, sanidine, J=0.0009783 $\pm 0.15 \%, D=1.0064 \pm 0.0005, N M-174 G, \quad$ Lab\#=54661 } \\
\hline \# & 03 & 14.458 & 1.150 & 6.364 & 0.941 & 0.444 & 87.651 & 22.247 & 0.257 \\
\hline \# & 08 & 13.898 & 1.242 & 3.474 & 0.773 & 0.411 & 93.354 & 22.774 & 0.291 \\
\hline \# & 12 & 14.841 & 1.244 & 6.397 & 0.570 & 0.410 & 87.957 & 22.912 & 0.368 \\
\hline \# & 05 & 16.102 & 1.806 & 10.742 & 1.038 & 0.283 & 81.215 & 22.963 & 0.267 \\
\hline \# & 13 & 14.216 & 1.355 & 3.943 & 0.521 & 0.377 & 92.593 & 23.105 & 0.346 \\
\hline \# & 11 & 13.566 & 1.154 & 1.624 & 0.563 & 0.442 & 97.167 & 23.135 & 0.350 \\
\hline \# & 07 & 14.252 & 1.119 & 3.514 & 1.246 & 0.456 & 93.364 & 23.352 & 0.250 \\
\hline \multirow[t]{5}{*}{ \# } & 09 & 13.429 & 1.085 & 0.637 & 0.599 & 0.470 & 99.268 & 23.394 & 0.355 \\
\hline & 06 & 13.587 & 0.010 & 0.207 & 13.060 & 52.474 & 99.556 & 23.717 & 0.178 \\
\hline & 04 & 13.731 & 0.008 & 0.566 & 11.359 & 60.036 & 98.787 & 23.783 & 0.178 \\
\hline & 14 & 13.733 & 0.009 & 0.484 & 12.209 & 57.403 & 98.963 & 23.829 & 0.177 \\
\hline & 10 & 14.056 & 0.011 & 1.494 & 8.885 & 45.706 & 96.866 & 23.873 & 0.183 \\
\hline \# & 01 & 14.708 & 1.361 & 3.500 & 1.237 & 0.375 & 93.735 & 24.192 & 0.240 \\
\hline \# & 02 & 14.254 & 1.276 & 1.397 & 1.228 & 0.400 & 97.845 & 24.470 & 0.239 \\
\hline \multirow[t]{2}{*}{ \# } & 15 & 13.871 & 1.347 & -1.205 & 0.311 & 0.379 & 103.371 & 25.155 & 0.709 \\
\hline & \multicolumn{2}{|c|}{ Mean age $\pm 2 s$} & $n=4$ & USWD $=0.14$ & & $53.9 \pm 12.6$ & & 23.799 & 0.192 \\
\hline
\end{tabular}




\section{Belleville Tuff}

\begin{tabular}{|c|c|c|c|c|c|c|c|c|c|}
\hline & \multicolumn{9}{|c|}{ ת } \\
\hline & 15 & 15.359 & 3.551 & 10.274 & 0.361 & 0.144 & 82.146 & 22.040 & 0.706 \\
\hline \multirow{13}{*}{ \# } & 10 & 48.182 & 3.457 & 119.110 & 1.245 & 0.148 & 27.543 & 23.172 & 0.483 \\
\hline & 14 & 17.984 & 3.471 & 16.515 & 0.275 & 0.147 & 74.461 & 23.382 & 0.797 \\
\hline & 01 & 36.404 & 5.645 & 79.367 & 0.594 & 0.090 & 36.859 & 23.464 & 0.747 \\
\hline & 06 & 15.532 & 3.368 & 7.710 & 0.528 & 0.151 & 87.125 & 23.625 & 0.507 \\
\hline & 02 & 22.248 & 3.972 & 30.164 & 0.440 & 0.128 & 61.413 & 23.861 & 0.754 \\
\hline & 11 & 15.008 & 2.539 & 5.080 & 0.840 & 0.201 & 91.397 & 23.932 & 0.362 \\
\hline & 03 & 14.112 & 2.534 & 2.029 & 0.622 & 0.201 & 97.237 & 23.940 & 0.518 \\
\hline & 04 & 18.523 & 2.069 & 16.213 & 0.657 & 0.247 & 75.060 & 24.247 & 0.542 \\
\hline & 13 & 15.291 & 3.630 & 5.491 & 0.754 & 0.141 & 91.354 & 24.387 & 0.398 \\
\hline & 12 & 17.299 & 3.198 & 11.719 & 0.546 & 0.160 & 81.510 & 24.607 & 0.552 \\
\hline & 07 & 23.507 & 5.243 & 33.336 & 0.607 & 0.097 & 59.939 & 24.624 & 0.455 \\
\hline & 09 & 18.196 & 3.363 & 14.424 & 0.374 & 0.152 & 78.106 & 24.803 & 0.648 \\
\hline & 05 & 15.638 & 6.630 & 6.489 & 0.461 & 0.077 & 91.247 & 24.960 & 0.713 \\
\hline \multirow[t]{2}{*}{ \# } & 08 & 16.974 & 2.926 & 7.177 & 0.173 & 0.174 & 88.932 & 26.325 & 1.119 \\
\hline & \multicolumn{2}{|c|}{ Mean age $\pm 2 s$} & $n=13$ & USWD=0.99 & & $0.15 \pm 0$ & & 24.089 & 0.294 \\
\hline
\end{tabular}

Metallic City Tuff (TT5)

\begin{tabular}{llllllllll}
\hline \multicolumn{7}{l}{ TT2d, sanidine, $\mathrm{J}=0.0010494 \pm 0.07 \%, \mathrm{D}=1.0064 \pm 0.0005, \mathrm{NM}-174 \mathrm{~F}, \mathrm{Lab}=54657$} \\
& 05 & 13.717 & 0.005 & 0.615 & 14.973 & 93.490 & 98.678 & 25.446 & 0.209 \\
& 06 & 14.625 & 0.007 & 3.614 & 13.112 & 69.334 & 92.701 & 25.487 & 0.209 \\
& 04 & 13.723 & 0.006 & 0.521 & 6.046 & 90.734 & 98.882 & 25.509 & 0.211 \\
& 09 & 13.699 & 0.009 & 0.397 & 6.513 & 57.568 & 99.148 & 25.533 & 0.212 \\
& 02 & 13.615 & 0.006 & 0.102 & 13.387 & 81.471 & 99.782 & 25.538 & 0.209 \\
& 03 & 13.800 & 0.006 & 0.697 & 13.306 & 90.330 & 98.512 & 25.556 & 0.208 \\
& 12 & 13.682 & 0.006 & 0.118 & 12.455 & 88.614 & 99.749 & 25.655 & 0.210 \\
& 07 & 13.669 & 0.007 & 0.055 & 5.811 & 74.581 & 99.884 & 25.664 & 0.213 \\
& 01 & 13.794 & 0.007 & 0.383 & 15.964 & 73.894 & 99.184 & 25.718 & 0.208 \\
& 08 & 13.789 & 0.007 & 0.321 & 12.048 & 70.128 & 99.317 & 25.742 & 0.209 \\
& 11 & 13.769 & 0.007 & 0.239 & 11.563 & 74.215 & 99.492 & 25.751 & 0.211 \\
& 15 & 13.777 & 0.007 & 0.172 & 10.607 & 69.102 & 99.636 & 25.803 & 0.209 \\
& 14 & 13.828 & 0.006 & 0.199 & 12.420 & 89.475 & 99.577 & 25.881 & 0.209 \\
& 10 & 16.931 & 5.536 & 9.422 & 0.302 & 0.092 & 86.262 & 27.547 & 0.809 \\
$\#$ & 13 & 14.445 & 4.605 & -4.032 & 0.157 & 0.111 & 110.886 & 30.169 & 1.663 \\
$\#$ & 13 & & & & 78.7 & \pm 22.2 & & 25.637 & 0.122
\end{tabular}




\section{Undivided Candelaria Sequence tuffs}

\begin{tabular}{|c|c|c|c|c|c|c|c|c|c|}
\hline & TT2 & hidine, & $=0.00106$ & $=0.07 \%, D$ & $0064 \pm 0$ & 05 , NM- & $\mathrm{F}, \mathrm{Lab} \#$ & 4658 & \\
\hline \# & 01 & 23.330 & 5.915 & 37.688 & 0.391 & 0.086 & 54.361 & 24.386 & 0.692 \\
\hline \# & 10 & 35.389 & 2.126 & 75.929 & 0.931 & 0.240 & 37.096 & 25.170 & 0.500 \\
\hline & 09 & 13.385 & 0.010 & 0.527 & 5.711 & 49.476 & 98.843 & 25.326 & 0.219 \\
\hline & 08 & 13.448 & 0.008 & 0.688 & 8.121 & 64.057 & 98.492 & 25.355 & 0.215 \\
\hline & 16 & 14.114 & 0.004 & 2.661 & 10.466 & 126.771 & 94.431 & 25.513 & 0.214 \\
\hline & 07 & 14.389 & 0.010 & 3.593 & 11.128 & 52.889 & 92.627 & 25.514 & 0.214 \\
\hline & 14 & 13.434 & 0.008 & 0.333 & 5.604 & 61.214 & 99.272 & 25.530 & 0.218 \\
\hline & 13 & 13.512 & 0.005 & 0.514 & 8.390 & 101.748 & 98.878 & 25.575 & 0.215 \\
\hline & 12 & 13.436 & 0.013 & 0.251 & 6.656 & 38.938 & 99.456 & 25.579 & 0.217 \\
\hline & 03 & 13.844 & 0.009 & 1.627 & 9.669 & 56.233 & 96.533 & 25.582 & 0.214 \\
\hline & 02 & 13.491 & 0.008 & 0.400 & 8.808 & 61.953 & 99.129 & 25.599 & 0.215 \\
\hline & 06 & 13.893 & 0.004 & 1.726 & 13.094 & 118.327 & 96.332 & 25.618 & 0.213 \\
\hline & 15 & 13.911 & 0.008 & 1.759 & 11.968 & 63.164 & 96.268 & 25.635 & 0.213 \\
\hline & 05 & 13.643 & 0.006 & 0.822 & 13.642 & 81.569 & 98.224 & 25.651 & 0.212 \\
\hline & 11 & 13.482 & 0.004 & 0.253 & 3.982 & 125.317 & 99.447 & 25.663 & 0.223 \\
\hline & 04 & 16.498 & 0.009 & 10.273 & 10.784 & 59.491 & 81.604 & 25.769 & 0.218 \\
\hline & $\mathrm{Me}$ & $e \pm 2 s$ & $n=14$ & USWD $=0.29$ & & $75.8 \quad \pm 59.5$ & & 25.565 & 0.12 \\
\hline & $\mathrm{TT}_{2}$ & sanidine, & $\mathrm{J}=0.0010$ & $\pm \pm 0.14 \%, D$ & -1.000 & $.0005, \mathrm{NM}-$ & 74G, Labł & $=54660$ & \\
\hline \# & 02 & 36.162 & 1.107 & 331.498 & 0.006 & 0.461 & -170.636 & -116.527 & $34.9 s$ \\
\hline & 11 & 15.134 & 0.007 & 1.409 & 9.894 & 72.782 & 97.254 & 26.697 & 0.18 \\
\hline & 10 & 15.003 & 0.008 & 0.912 & 9.171 & 66.844 & 98.207 & 26.725 & 0.185 \\
\hline & 03 & 15.235 & 0.008 & 1.698 & 5.843 & 61.661 & 96.711 & 26.726 & 0.190 \\
\hline & 04 & 14.895 & 0.008 & 0.476 & 17.066 & 65.708 & 99.060 & 26.763 & 0.186 \\
\hline & 15 & 17.060 & 0.009 & 7.629 & 10.173 & 59.326 & 86.790 & 26.856 & 0.192 \\
\hline & 13 & 15.074 & 0.008 & 0.880 & 9.538 & 67.293 & 98.279 & 26.870 & 0.18 \\
\hline & 12 & 15.157 & 0.008 & 1.040 & 11.624 & 66.897 & 97.977 & 26.934 & 0.18 \\
\hline & 06 & 15.010 & 0.007 & 0.542 & 12.515 & 74.610 & 98.937 & 26.935 & 0.18 \\
\hline & 09 & 15.042 & 0.007 & 0.620 & 9.468 & 69.547 & 98.785 & 26.951 & 0.18 \\
\hline & 07 & 14.882 & 0.008 & 0.077 & 8.020 & 65.284 & 99.852 & 26.953 & 0.18 \\
\hline & 08 & 14.929 & 0.008 & 0.215 & 8.923 & 64.564 & 99.578 & 26.963 & 0.18 \\
\hline & 05 & 15.020 & 0.008 & 0.505 & 16.508 & 60.755 & 99.012 & 26.973 & 0.18 \\
\hline & 01 & 15.036 & 0.008 & 0.457 & 14.675 & 64.808 & 99.106 & 27.026 & 0.18 \\
\hline & 14 & 15.085 & 0.007 & 0.281 & 9.654 & 75.284 & 99.453 & 27.208 & 0.18 \\
\hline & $\mathrm{Me}$ & $e \pm 2 s$ & $n=14$ & USWD $=0.56$ & & $66.8 \pm 9.7$ & & 26.900 & 0.12 \\
\hline
\end{tabular}




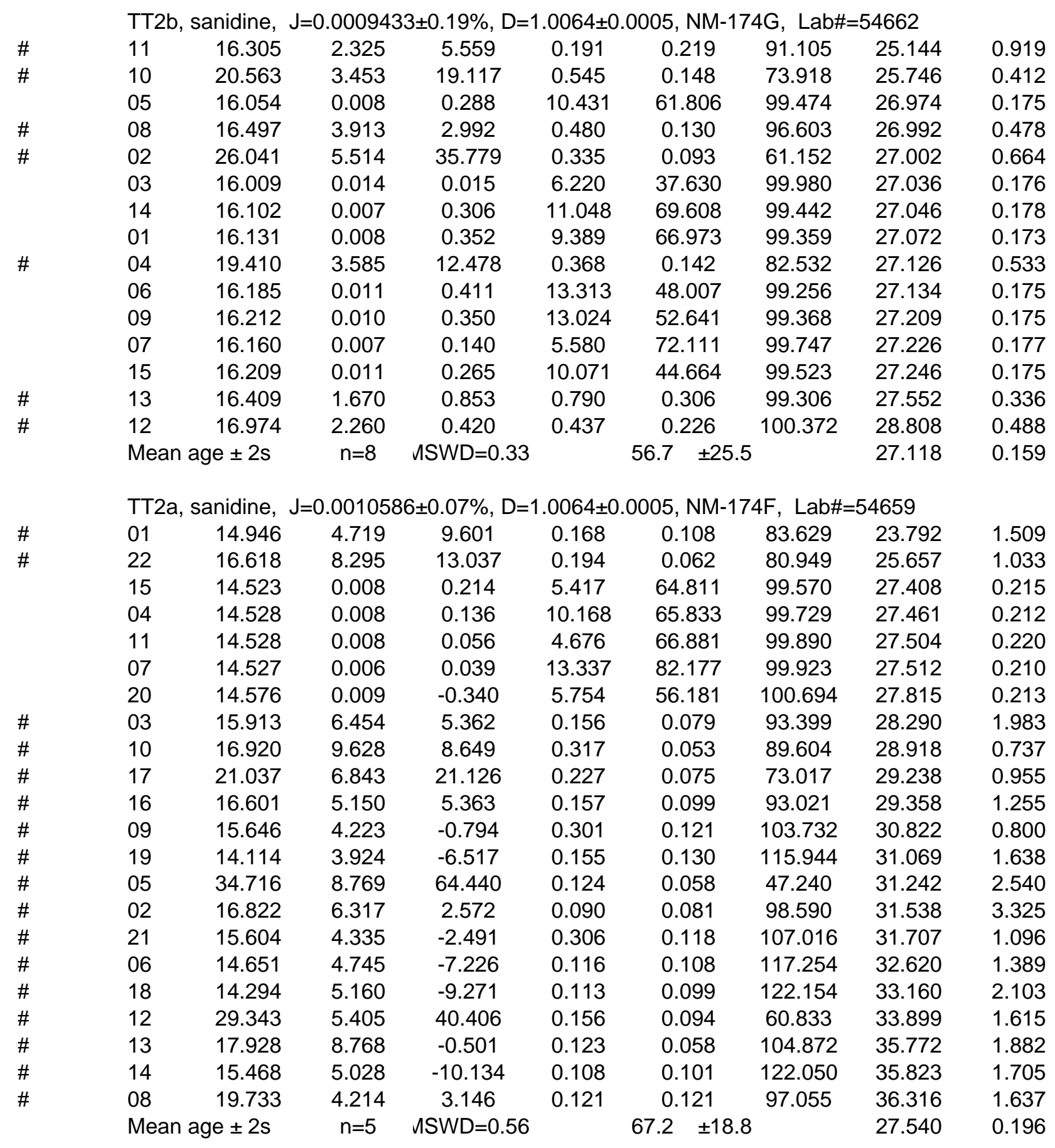


Notes:

Isotopic ratios corrected for blank, radioactive decay, and mass discrimination,

Ages calculated ralative to FC-1 Fish Canyon Tuff sanidine interlaboratory standard at $27.84 \mathrm{Ma}$. not corrected for interferring reactions. Errors quoted for individual analyses include analytical error only, without interferring reaction or J uncertainties.

Mean age is weighted mean age of Taylor (1982). Mean age error is weighted error

of the mean (Taylor, 1982), multiplied by the root of the MSWD where MSWD>1, and also incorporates uncertainty in $\mathrm{J}$ factors and irradiation correction uncertainties.

Decay constants and isotopic abundances after Steiger and Jaeger (1977).

\# symbol preceding sample ID denotes analyses excluded from mean age calculations.

Discrimination $=1.0064 \pm 0.0005$

Correction factors:

(39Ar/37Ar) $\mathrm{Ca}=0.0007 \pm 5 \mathrm{e}-05$

$(36 \mathrm{Ar} / 37 \mathrm{Ar}) \mathrm{Ca}=0.00028 \pm 1 \mathrm{e}-05$

(38Ar/39Ar)K $=0.0129$

(40Ar/39Ar)K $=0 \pm 0.11$ 